\title{
Strategies to Cope with Moral Distress among Mental Health Nurses in Thailand
}

\author{
Ratchaneekorn Upasen, Ph.D. ${ }^{1}$, Weeraphol Saengpanya, Ph.D. ${ }^{2}$, Juraipon Sambutthanon, R.N. ${ }^{3}$ \\ ${ }^{1}$ Faculty of Nursing, Chulalongkorn University, Pathumwan, Bangkok 10330, Thailand. \\ ${ }^{2}$ Faculty of Education, Chulalongkorn University, Pathumwan, Bangkok 10330, Thailand. \\ ${ }^{3}$ Faculty of Nursing, Khon Kaen University, Mueang, Khon Kaen 40002, Thailand. \\ Received 31 March 2020 • Revised 10 May 2020 • Accepted 16 May 2020 • Published online 14 September 2020
}

\begin{abstract}
Objective: The purpose of this study was to explore the strategies used by Thai mental health nurses to cope with the moral distress that often results from their work.

Material and Methods: The study was performed using a qualitative, narrative method. Participants were recruited into the study using a purposive snowball method. A total of 41 mental health nurses met the inclusion criteria and were in-depth interviewed about their strategies to cope with moral distress, with content analysis being used to analyze the data.

Results: Six principal themes were found, classified as (1) releasing tension by talking to others, (2) positive self-talk, (3) believing in my ability to handle problems, (4) using my training and experience of nursing to self-help, (5) being with professional autonomy, and (6) participating in a case conference group. These strategies were used by the Thai mental health nurses in the study to cope with the moral distress resulting from their work.

Conclusion: Awareness of these strategies to cope with the moral distress related to work can benefit mental health nurse leaders, including head nurse and head ward nurses to provide suitable nursing staff development to enhance the quality of the psychiatric nursing practice.
\end{abstract}

Keywords: coping, distress, mental health, moral, nurses

Contact: Weeraphol Saengpanya, Ph.D.

Faculty of Education, Chulalongkorn University, Pathumwan, Bangkok 10330, Thailand.

E-mail: weeraphol.s@chula.ac.th, createpanya@hotmail.com

() 2020 JHSMR. Hosting by Prince of Songkla University. All rights reserved.

This is an open access article under the CC BY-NC-ND license

(http://www.jhsmr.org/index.php/jhsmr/about/editorialPolicies\#openAccessPolicy).
J Health Sci Med Res 2021;39(1):47-55 doi: 10.31584/jhsmr.2020762 www.jhsmr.org 


\section{Introduction}

Mental health nurses (MHNs) work with patients who struggle with severe psychotic disorders in the acute, sub-acute, and rehabilitation phases. ${ }^{1-3}$ They may face ethical dilemmas and moral distress during caring for patients with mental illnesses and their families. Moral distress can happen when a nurse cannot take a moral action even though they know how to take the appropriate moral action to protect or help their patients, but they cannot take the action because of various barriers. ${ }^{4-6}$ There are a variety of moral dilemma situations that can lead to moral distress for MHNs during their work every day, such as the use of restraints, nursing assistance for electro-convulsive therapy, separating a patient into a seclusion room, compulsory admission, participating in group therapy, and administering medication to incompliant patients. ${ }^{4}$ One study found that 202 MHNs believed that they had committed unethical behaviors due to various internal and external constraints while providing care for patients with mental illness, including neglect, careless behaviors, disrespect of the patient's rights and human dignity, bystander apathy, lack of appropriate physical treatments during restraint, interfering with a patient's of patient's privacy, lack of clarity, not asking for permission, and exposure to physical illness. ${ }^{5}$ When such actions occur, the quality of care and patient satisfaction may be affected. ${ }^{5,6}$ In addition, staff retention can be low and staff shortages high ${ }^{6,7}$, as reported in a study by Nathaniel ${ }^{8}$ which concluded that an important consequence of moral distress is the change of employment from one workplace to another one, often in a different field. Previous research in the general nursing context has reflected both negative and positive experiences and consequences of ethical problems, including moral distress. Some studies have found that MHNs experienced moral distress. ${ }^{9-11}$ For instance, a research survey about moral distress in 228
$\mathrm{MHNs}$ found that the median moral distress level was 2 out of $6 .{ }^{11}$

Previous studies have explored moral distress among health professionals in several nursing fields, including critical care, emergency care, and mental health settings. ${ }^{12-16}$ These studies found that most nurses experienced moral distress in caring for their patients and the patients' families, as characterized by feelings of selfdoubt, guilt, frustration, anger, depression, and stress. ${ }^{15,17,18}$ Another study on moral distress in 12 registered psychiatric nurses working with adolescents in local communities found that most of the registered psychiatric nurses had feelings of inability and uncertainty over maintaining the patients' safety, changing jobs, and conflicts with the expectations of co-workers and mental health agency policy. ${ }^{13}$ Other studies found that the negative consequences of moral distress on the psychological health of nurses included sadness about being unable to respond adequately to patients' needs; feelings of not being safe in their workplace, disrespect from patients and families, and serious institutional constraints, all related to personal and professional distress as well as lack of connection with co-worker. ${ }^{5,6,9}$ These previous studies focused only on understanding nurses' experiencing moral distress. ${ }^{15,17,18}$ However, few previous studies have explored strategies to cope with moral distress among MHNs, reporting only the specific characteristics of moral distress in MHNs.

Moral distress is recognized as an important factor in high nursing shortage and turnover rates. ${ }^{19}$ Nursing leaders should recognize its presence and take a role to advocate measures to help nurses to cope with the moral distress that often accompanies their work. ${ }^{20}$ However, little research has been conducted on strategies to cope with moral distress in mental health settings. In Thailand, there have been no studies on strategies to cope with moral distress in mental health nursing. Identifying and 
understanding such strategies should be basic knowledge for mental health professionals, including nurses, so they can apply these strategies when they face moral distress issues or to use in planning approaches to mitigate moral distress. Positive consequences can result from such strategies, such as providing improved quality of mental health nursing and good care of the patients by the MHNs. ${ }^{18,20}$ Furthermore, mental health organizations may use the knowledge to set up mental health policies to decrease the problem of serious shortages of MHNs and staff at psychiatric hospitals, in common with other countries. ${ }^{21,18}$ This research aimed to explore strategies adopted by Thai MHNs to cope with their moral distress.

The purpose of this study was to explore the strategies used by Thai mental health nurses to cope with moral distress.

\section{Material and Methods}

This article is a part of a narrative research titled: A narrative research on moral distress in mental health nursing practice. This research was approved by the Health Ethics Review Board of Chulalongkorn University (Protocol No. 183.1/60) and by The Ethical Review Committee for Research in Human Subjects of four psychiatric hospitals in Thailand. To better understand the strategies used by Thai MHNs to cope with moral distress, a narrative method $^{22}$ was used in this study. The narrative method invites the MHNs to talk about times when they were faced with moral distress during work when caring for patients with mental illness.

The participants in this study were $41 \mathrm{MHNs}$ who were working in four psychiatric hospitals in Thailand. Purposive sampling and the snowball technique were used to recruit the participants. The inclusion criteria were: (1) MHNs who had been employed in a psychiatric hospital for at least the previous 2 years, (2) MHNs who could verbally share information about coping with moral distress, and (3) willing and able to participate in the research. The potential participants in this study received research information, including the purpose, benefits and risks of the study, and the right to withdraw from the study without a given reason. After the potential participants signed the informed consent form, the researcher made an appointment to conduct the interview. The 41 participants were comprised of 34 females and seven males. For their highest education level, 21 participants had a Bachelor's degree and 20 participants had a Master's degree. Their ages ranged from 27-52 years and their mental health nursing experience ranged between 2.5-21 years.

The first researcher conducted a semi-structured face-to-face interview with each participant, which took approximately 45-60 minutes, in a private room in the selected psychiatric hospital. Major open-ended questions, such as "what are the strategies that you use to cope with moral distress?" and "how do you use the strategies to be successful?" were asked. All interviews were audiotaped and transcribed verbatim. In this study, the data saturation refer to there are no new information in data analysis was obtained from the $41 \mathrm{MHNs}$ and no participants dropped out.

We analyzed the data simultaneously alongside data collection. Content analysis was used to find strategies that the MHNs used to cope with moral distress. All transcription were checked by the researchers. The researchers then read each transcription carefully and text-highlighted those parts relating directly to using strategies to cope with the experience of moral distress. The researchers selected the content from each transcript and defined the categories of analysis. The researchers then developed a set of rules for coding and coded the text according to the rules. Once the coding was complete, 
the collected data were examined to find any patterns of coping and conclusions were drawn in response to the research objective. The patterns of coping included methods for releasing tension by talking to others, (2) positive self-talk, (3) believing in my ability to handle problems.

The four criteria of Reissman ${ }^{22}$ to enhance the trustworthiness of narrative research were applied: (1) the narratives about strategies of coping with moral distress are supported by the participant's account; (2) the researchers returnedtranscription to validate and confirm the meaning of the participant; (3) the researchers looked for consistent relationship among categories of coping strategies; and (4) the knowledge about strategies to cope with moral distress used by MHNs can be applied to decrease and prevent moral distress in other clinical settings. In addition, the first researcher used dependability, which is an important method for keeping rigor in all processes of this study by discussing with co-researchers about recruitment the participants, data collection, and data analysis of this study.

\section{Results}

The participants (MHNs) in this study shared their strategies to cope with moral distress they experienced during mental health nursing. There were six principal themes that describe their strategies, as discussed in turn below.

\section{Releasing tension by talking to others}

One strategy used by all MHNs (41/41; 100.0\%) in this study was to share their experiences regarding their moral distress with others, including talking to health care team members, ward heads, and close friends or families about how to deal with distressing situations and what happened in the situation. While the participants talked to family members such as their spouse, they did not expect to get a solution (ways to deal with the problem), but rather they just wanted to release tension. In explaining the problem/situation to the other person they had to think about the problem(s) and why they felt bad or guilty. The participants also talked to their ward head and coworkers on how to solve their distress problems and how to manage their jobs. After they talked to the health care team members, ward heads, and close friends or family members, they were more comfortable and had ways to deal with the problems and to care for the patients. Two examples are given below:

"When I am uncomfortable, stressed, and sad, I always talk to my head about what is going on, and what I should do with the situation." (Case 27, page 5, lines 10-11).

"For me, I like talking to my family even if they do not clearly understand the situation and they listen to me and support my feelings. I also talk to my colleagues about how I was feeling and they always mentally support me." (Case 28, page 2, lines 26-28).

\section{Positive self-talk}

In another approach, some of the MHNs (32/41; $78.0 \%$ ) have used a strategy of positive self-talk to support themselves when they experienced symptoms of moral distress. With the strategy, the participant would review what had happened and look for the causes of the negative feelings to understand them by themselves. Another related measure was positive self-talk, such as telling themselves that they had done the best they could for their patient's safety. The positive self-talk helped the MHNs feel better about themselves and the things that were going on in their lives, as well as reducing their stress. This is exemplified by the comments of two participants below: 
"I understand my own emotions more clearly and by reviewing what is going on about myself by doing selftalk. As a MHN, I am proud to be a MHN. I am supposed to understand the symptoms of the patients and calm my feelings down. I do everything the best to keep my patients safe." (Case 36, page 2, lines 7-9).

"At that time, I could not sleep. I thought that was my fault that the patient escaped from the hospital. I also had anxiety about the patient's safety. In my life, I always think a lot. I try to think positively and self-talk to myself that I do good things for my patients. I do my best. Then I am comfortable." (Case 27, page 1, lines 20-23).

\section{Believing in my ability to handle problems}

Believe myself to diminishing the problems is another strategy used by all MHNs $(41 / 41 ; 100.0 \%)$ to cope with their moral distress. When the participants were faced with moral distress. they force themselves to think that the problem is not so big that they cannot deal with it, that they are capable of dealing with this problem. They can solve the problems by themselves or ask for help from a peer, boss, or family. The participants also used a strategy of diminishing the importance of the problem to cope with moral distress. Diminishing the problem is a way of making the problem to be less important. The participants had an understanding and perceived that the problems could be solved. After they diminished the problem in their minds, they felt more comfortable. They also looked for other ways to cope with the distressing situation. As these two participants said:

"I know that I am dealing with the problem. I try to think it's alright. I have my boss and my friends to consult with. I think that the distressful situation will be gone soon. However, I also give the good care for the patients." (Case 3, page 5, lines 17-19).
"Sometimes, when I feel stress with my job, especially, I feel guilty about that. I try to think what happened. I could not change anything. Everybody does that, it's not only me. So, I try to tell myself that it's a little thing. It's a problem in my life. I can resolve it." (Case 1, page 6, lines 15-20).

\section{Using my training and experience of nursing to} self-help

Using their knowledge and experience to self-help is a strategy that all MHNs $(41 / 41 ; 100.0 \%)$ used to cope with their moral distress. When the participants felt frustration, stress, or guilt, they always thought that they are MHNs who have mental health knowledge. They can apply their knowledge to care for themself. The MHNs used a self-therapeutic approach, which is a valuable instrument in caring for the patients. Accordingly, the MHNs were aware of their own thoughts, feelings, and actions while caring for the patients in order to better understand the patient and the patient's family. The MHNs, in this study, tried to understand what they did and how they were feeling. At the same time, they understood the patients. In addition, the participants re-thought their roles and tried to use their knowledge, such as cognitive behavioral therapy, to help themselves to adjust their thinking to be positive when facing problems. This is exemplified by the participant' comments below:

"I am angry; I know my feeling. I try to control my own feelings. I am aware about my speaking or talking when I meet patients and families. It's the role of being a MHN that I used. I do meditation and try to think carefully before doing everything." (Case 38, page 3, lines 5-9).

"When I feel sad or be stressed, I use my knowledge about this to treat myself. I try to think of patients just 
as patients. I think that I can bring my knowledge to selfhelp when I suffer from the distress." (Case 29, page 1, lines 26-27).

\section{Being with professional autonomy}

Being with professional autonomy is another strategy that all MHNs $(41 / 41 ; 100.0 \%)$ used to cope with their moral distress. Professional autonomy is defined as the capacity of a person to act in accordance with objective morality and professional knowledge base rather than under the influence of desires. For instance, the participants used their own autonomy to decide whether or not to restrain patients who were showing aggressive behaviors before receiving the treatment plan from the psychiatrist in order to protect the patients from harm. The MHNs understand the problem of moral dilemma while caring for their patients. According to the MHNs' autonomy, the participants also used selfdirecting freedom and, especially, moral independence and personal and professional autonomy while giving care for their patients or dealing with problems. At such times, the MHNs were aware of the patient's human rights to be free and not forced. As one participant said:

"When I feel bad, I know my feelings. I rethink about my role as a MHN. I try to care for the patient's right under my responsibility to be safe. I decide to restrain the patient to protect the patient and other people around them, even if the patient and family disagreed with the care. I thought that what I did was good for the patient's safety." (Case 4, page 6, lines 7-10).

\section{Participating in a case conference group}

A case conference group is an important approach where the participants can share their experiences in caring for patients. About half $(22 / 41 ; 53.7 \%)$ of the MHNs in this study used this participation to find ways to deal with problems from the multidisciplinary team members who had experience in caring for patients. The participants shared their distressing situation(s), including their feelings/Impacts in the group. Also, these meetings offer good opportunities to suggest the need to review and revise the caring procedures for patients so as to improve them and have a higher quality of care. Moreover, the participants felt that thes groups could help them to be comfortable and provide guidelines to confront their problems. As two articipants said:

"It's a good time that I can share my experience in caring for patients. I think that in the group, we talk together about the causes of problems. Members in the group do not mention individual errors, but we talk about how to solve and prevent the problems. I feel good and calm down. I can care for the other patients much better" (Case 7, page 5, lines 2-6).

"In the case conference, team members talk about how to plan for helping patients and families. I think that these meetings help me learn how to deal with the problems and how to care for the next patients. The case conference group should be held every week or month. Every body in the team should talk together about caring for the patients" (Case 14, page 3, lines 11-18).

In this study, six strategies used by the MHNs to cope with their moral distress were discussed, namely releasing tension by talking to others, exercising positive self-talk, //believe myself to diminishing the problems//, using knowledge to self-help, autonomy, and participating in a case conference group. While some of the participants used only one, two, or three of these strategies, some used five or all six of them to cope with their moral distress, depending on individual differences and the support needed (Figure 1). 


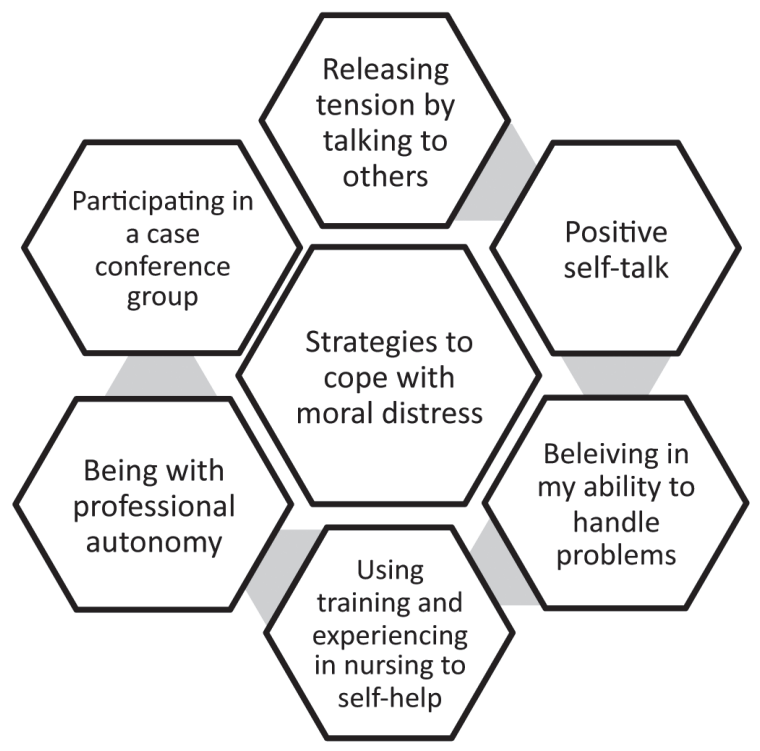

Figure 1 Strategies to cope with moral distress of mental health nurses

\section{Discussion}

This research found that the MHNs who participated in this study had developed important strategies for coping with the moral distress in caring for their patients by themselves. Most of the participants used several strategies to cope with their moral distress. The most common strategy (41 cases) used was talking with someone to relieve their tension, including talking with the head ward, co-workers, peers, and family members. In the talks, the participants felt more comfortable after having someone listen to them and their negative feelings were released. The participants also had greater knowledge of the details of the distressing situation after talking with the team members or co-workers and after participating in a conference group. In the same way, the participants learned about different choices and ways to deal with their problems, including negative feelings such as fear, guilty, and sadness after they coped with the moral distress. Furthermore, talking with others encouraged the participants to look for good ways to care for their patients. This finding is consistent with previous studies that have shown similar results - that nurses who are experiencing moral distress often use sources of support from peers, teamwork, family members, and hospital managers $^{13,15,23,24}$ Consequently, the MHNs felt that they were not alone while dealing with problems. ${ }^{13}$

A positive self-talk strategy was used by the MHNs to confront their moral distress. They reported that using positive self-talk decreased their moral distress and that it was a good way to help them feel better. Positive self-talk can affect the participants' perspective and boost them to reach their nursing goals. They always think that they provide good care for their patients. This finding is consistent with a study conducted by Musto and Schreiber ${ }^{13}$ about exploring the processes used by mental health nurses who experienced moral distress in working with adolescents. Their study found that shifting the perspective of the MHNs is a stage in dealing with moral distress. That means that after such talks, the MHNs had better perspectives in terms of understanding their actions and had an answer for the question "Is this the best I can do?" and continued their work with the adolescents. At the same time, the MHNs used the strategy of believing in my ability to handle problems to cope with their moral distress. This strategy is recognized as an emotionfocused coping way. ${ }^{25}$ When the participants experienced moral distress, such as stress, anxiety, or a feeling of guilt, they used this strategy to diminish the importance of the problem to become less important. Also, they used other strategies, including releasing tension, being with professional autonomy, and using my training and experience of nursing to self-help to reduce their moral distress. However, one thing that is important is the participants must perceive that they have more choices and support resources when they use this strategy.

Being with professional autonomy is another strategy employed by the MHNs to help them cope with 
their moral distress. When the participants provide care for patients or are involved with the patients' families, they use this strategy by giving care for patients under their independent nursing roles and accountability, and so they can cope with their moral distress themselves. This finding is consistent with a study by Lievrouw et al. ${ }^{26}$, who that found that this approach was used by oncology practitioners to cope with their moral distress, as well as another study that reported that nurses who have professional autonomy are associated with lower levels of moral distress ${ }^{27}$ since the strategy makes the nurses confront their moral distress. This finding reflects the power of autonomy used by the MHNs to reduce their moral distress.

Another strategy used to cope with moral distress by the MHNs was to use their training and experiencing in nursing to self-help. The participants (41 cases) shared that they used their knowledge on stress management, interpersonal relationships, and communication techniques for self-help when confronted with moral distress, since they have learned how to mentally support their patients. This knowledge can be conceptual frameworks to solve problems, especially with coping with their moral distress. At the same time, the MHNs participated in case conference groups, which allow the health care providers to discuss about any situation and how to solve the problems. This strategy can benefit the MHNs because they have time to find good ways to deal with distressful situations and cope with the problems. They also have a good way to review the nursing procedures in order to provide a better quality of care.

\section{Conclusion}

Strategies to cope with moral distress should be employed at the beginning of the situation in order to protect MHNs from moral distress. Strategies adopted by Thai MHNs to cope with their moral distress include releasing tension by talking to others, positive self-talk, believing in my ability to handle problems, using my training and experience of nursing to self-help, being with professional autonomy, and participating in a case conference group. The MHNs and other health care professionals can apply these strategies to reduce their moral distress when faced with distressful situations in clinical practice. It would be interesting and informative to see future studies explore the factors related to or predicting the factors of moral distress, as well as the development of nursing interventions to help MHNs cope with moral distress.

\section{Acknowledgement}

The authors are especially grateful to the psychiatric nurses who participated in this study. The graduate school of Chulalongkorn University is acknowledged for supporting a research assistant to conduct the research.

\section{Funding sources}

This research was supported by the Ratchadaphiseksomphot Endowment Fund of Chulalongkorn University.

\section{Conflict of interest}

No conflicts of interest have been declared by the authors.

\section{References}

1. Sadock BJ, Sadock AV. Comprehensive textbook of psychiatry. Philadelphia: Lippincott, Williams and Wilkins; 2017.

2. Moe C, Kvig El, Brinchmann B, Brinchmann Bs. "Working behind the scenes". An ethical view of mental health nursing and first-episode psychosis. Nurs Ethics 2013;20:517-27.

3. Weimand BM, Sallstrom C, Hall-Lord ML, Hedelin B. Nurses' dilemmas concerning support of relatives in mental health care. Nurs Ethics 2013;20:285-98. 
4. Choe $\mathrm{K}$, Song $\mathrm{EJ}$, Jung $\mathrm{CH}$. Ethical problems experienced by psychiatric nurses in Korea. Arch Psychiatr Nurs 2012; 26:495-502.

5. Eren N. Nurses' attitudes toward ethical issues in psychiatric inpatient settings. Nurs Ethics 2013. doi: 10.1177/ 0969733013500161.

6. Mekechuk J. Moral distress in the pediatric intensive care unit: the impact on pediatric nurses. Int $\mathrm{J}$ Health Care Qual Assur Inc Leadersh Health Serv 2006;19:i-vi.

7. Gutierrez K. Critical care nurses' perceptions of and responses to moral distress. Dimens Crit Care Nurs 2005;24: 229-41.

8. Nathaniel A. Moral reckoning in nursing. West $\mathrm{J}$ Nurs Res 2006;28:419-38.

9. Shaher H. Moral distress and its correlates among mental health nurses in Jordan. Int J Ment Health Nurs 2014;23:33-41.

10. Jansen TL, Hanssen I. Patient participation: causing moral distress in psychiatric nursing?. Scand J Caring Sci 2016;388: 94.

11. Delfrate F, Ferrara P, Spotti D, Terzoni S, Lamiani G, Canciani $\mathrm{E}$, et al. Moral distress (MD) and burnout in mental health nurses: a multicenter survey. Med Lav 2018;109:97-109.

12. Powell SB, Engelke KM, Swanson MS. Moral distress among school nurses. J Sch Nurs 2018;34:390-7.

13. Musto L, Shreiber RS. Doing the best I can do: moral distress in adolescent mental health nursing. Issues Ment Health Nurs 2012;33:137-44.

14. Austin W, Rankel M, Kagan, L, Bergum V, Lemermeyer G. To stay or to go, to speak or stay silent, to act or not to act: moral distress as experienced by psychologists. Ethics Behav 2005;15:197-212.

15. Deady RM, McCarthy J. A study of the situation, features, and coping mechanisms experienced by Irish psychiatric nurses. Perspect Psychiatr Care 2010;46:209-20.
16. Kirkpatrick H. A narrative framework for understanding experiences of people with severe mental illness. Arch Psychiatr Nurs 2008;22:61-8.

17. Hall LM, Kiesners D. A narrative approach to understanding the nursing work environment in Canada. Soc Sci Med 2005;61:2482-91.

18. Austin W, Bergum V, Goldberg L. Unable to answer the call of our patients: mental health nurses' experience of moral distress. Nurs Inq 2003;10:177-83.

19. Corley MC. Nurse moral distress: a proposed theory and research agenda. Am J Crit Care 1995;4:280-5.

20. Bell J, Breslin JM. Healthcare provider moral distress as a leadership challenge. JONAS Health Law Ethics Regul 2008;10:94-7.

21. Hanrahan N. Psychiatric nurse staffing in hospitals: is it adequate?. J Am Psychiatr Nurses Assoc 2012;18:27-9.

22. Riessman CK. Narrative methods for the human sciences. London: SAGE; 2008.

23. Maluwa VM, Andre J, Ndebele P, Chilemba E. Moral distress in nursing practice in Malawi. Nurs Ethics 2012;19:196-207.

24. Kertchok R. Ethical issues and moral distress in psychiatric and mental health nursing: a literature review. J Health Res 2015;29:227-34.

25. Lazarus RS, Folkman S. Coping and adaptations. In: Gentry WD, editors. Handbook of behavioral medicine. New York: The Guildford Press; 1984:p.282-325.

26. Lievrouw A, Vanheule S, Deveugele M, De Vos M, Pattyn P, Belle V. et al. Coping with moral distress in oncology practice: nurse and physician strategies. Oncol Nurs Forum 2016;43: 505-12.

27. Sarkoohijiabalbarezi Z, Ghodovsi A, Davaridolatabadi E. The relationship between professional autonomy and moral distress among nurses working in children's units and pediatric intensive care wards. Int J Nurs Sci 2017;4:117-21. 